fact that animals possess faculties differing from those of man is an insuperable obstacle to a perfect analysis of their intelli. gences.

Name these faculties as you please, call them "inherited habit," "inherited memory," it is perfectly certain that man does not possess them.

H. D. BARCLAY

\section{WILLIAM FROUDE}

THE death of Mr. W. Froude, F.R.S., is a loss to science that cannot well be estimated. For many years he laboured with great ability and success in a field of research that was beset with difficulties, and had previously been almost barren of results. He was educated at Westminster, and went from there to Oxford, where he distinguished himself in mathematics. After leaving Oxford he became a civil engineer, and assisted Mr. Brunel in railway and other engineering work. He retired from active professional life in 1846 , but his love of applied science retained such a hold upon him that he never ceased to occupy himself with important scientific investigations, and the solution of practical problems of peculiar difficulty. His intimacy with $\mathrm{Mr}$. Brunel led to his mind being directed towards the study of those laws of nature which govern the motion of floating bodies. Mr. Brunel had devoted himself, among other things, to the improvement and development of iron steamship construction. In the Great Western and Great Britain he had made great advances in this direction; while in the Great Eastern he showed that iron and steam power could be employed in the production of ships of practically unlimited dimensions, and that by means of these agencies all the advantages appertaining to increased size might be realised.

In designing ships of such exceptional character and dimensions, Mr. Brunel found little to guide him in judging of their behaviour at sea. They were so different to any vessels afloat whose behaviour and qualities might have been ascertained, that he was unable to appeal to experience, while the light of science was so feeble and doubtful as to afford him no aid. Nobody at that time knew anything of the laws upon which a ship's motion at sea depends. There was a large mass of traditional experience, but this was often at variance with fact, owing to phenomena which are familiar to seamen being regarded as absolute, and possessing a reality of existence as well as of appearance; while, as must be obvious, they are only relative in their character, and cannot be accurately defined without making due allowance for the position and motion of a ship, with reference to the sea. The rules and maxims that had been adopted upon such incorrect and distorted data, were either unimportant or misleading; they were of no value. Mr. Froude said, quite correctly, in I86I, that our shipbuilders, while extending their knowledge in other directions, seem to have guided themselves by rhetorical phrases or random speculations in this particular branch of their art, "so that when a new ship is sent to sea, her constructor has to watch her behaviour in a seaway, with as anxious and uncertain an eye as if she were an animal he had bred and was rearing, and hoped would turn out well, not a work which he had himself completed, and whose performance he could predict, in virtue of the principles he had acted on in its design."

Mr. Froude, at the request of Mr. Brunel, commenced in 1856 an investigation into the laws of motion of a ship among waves. This had been previously attempted by D. Bernouilli, Euler, Moseley, and others, but without success. None of these writers had realised the fundamental conditions of the action of wave-water upon a ship, viz., that the direction and intensity of the fluidpressure at any point is continually changing, and that the direction of pressure is normal to the surface of equal pressure passing through that point. They based their theories upon hypotheses respecting wave-action that were all more or less erroneous, and prevented any useful result being realised. Mr. Froude's method of dealing with the subject was, first of all, to determine the manner in which a wave acts upon a ship; or, in other words, the mode of operation of the agency whose effects he wished to comprehend. In this he was completely successful, and proved in an unexceptionable manner the mechanical possibility of that form of motion known as the trochoidal sea-wave. On the assumption that the motion of each particle on the surface of a wave describes an exact circle, whose diameter is the height of the wave from hollow to crest-which agrees with the results of observation-and that the motions of all particles lie in vertical planes which cut the wave-ridges at right angles, he deduced the theory that the form of the wave would be trochoidal, and that the periodic time would be equal to the time occupied by a heavy body in falling through a height equal to the circumference of a circle whose diameter is the length of a wave. It also followed that all sub-surfaces of equal pressure would be trochoids of the same length as the surface-wave, but of a height which would diminish with the depth in accordance with the equation $\frac{r_{0}}{r_{d}}=e \frac{\pi}{\mathrm{L}} d$, where $e$ is the base of Napierian logarithms, $L$ the length of the wave from hollow to crest, $d$ the depth of the centre of the circle described by any particle below that of the circle described by the surface-particle, $r_{d}$ the radius of the circle at the depth $d$, and $r_{0}$ that of the circle at the surface. Prof. Rankine also independently deduced the same theory. A striking feature of the investigation was the rapid decrease in the motions of the particles as they are traced to lower depths. Prof. Stokes showed that for all waves of ordinary proportions, the motion at a depth equal to the length of the whole wave from crest to crest is only $\frac{1}{55}$ of that which belongs to a surface particle. The dynamical conditions of wave-water being thoroughly investigated and established, Mr. Froude next proceeded to base upon it a scientific theory of the rolling of ships among waves.

The subject was first brought before the public by Mr. Froude in a paper read before the Institution of Naval Architects in $186 \mathrm{r}$. He stated that he felt some diffidence in bringing forward "what assumes to be a tolerably complete theoretical elucidation of a difficult and intricate subject, which has hitherto been treated as if unapproachable by the methods of regular investigation." He pointed out that the characteristicfea ture of the dynamical laws to which it would be necessary to refer the movements of a ship when rolling is the gradual accumulation of angle during several successive rolls, the cumulative action thus growing up into a maximum, and then dying out by very similar gradations until the ship becomes for a moment steady, when a nearly isimilar series of excursions commences and is reproduced; while in reference to the momentary pause, or cessation of motion, it seems clear that it occurs, not because the waves themselves cease, or cease to act, but because the last oscillation has died out at a moment when the ship and the waves bave come to occupy, relatively, a position of momentary equilibrium. This is so closely analogous to what happens when a pendulum is subjected to a series of impulses, partially synchronous with its own excursions, that it seemed probable that the laws which govern the latter class of phenomena would be found, mutatis mutandis, applicable to the elucidation of the former also. The investigation of the laws of rolling motion, when thus regarded, therefore assumed the form of the inquiry, "What is the cumulative result of the continuous action of a series of consecutive wares operating on a given ship?"

In order to determine this it was necessary first to determine how each individual wave will act upon a ship at each instant of time; or, in other words, "What is the position of momentary equilibrium for a body floating on a wave, and what accelerating force towards that position 
will the body experience in terms of her momentary deviation from it?" Mr. Froude has demonstrated both $\dot{a}$ priori and experimentally that to a stabilised particle floating at any point on the upper surface of a wave, the position of momentary equilibrium is that which would place the axis of equilibrium normal to the wave surface at the point where it floats, and that to another similar particle, floating or suspended below the surface, the position of momentary equilibrium is that which would place the axis normal to the sub-surface of equal pressure passing through the point where it is placed. If we take account of the aggregation of particles which a ship displaces, and for which she herself is substituted, and of which she accepts the aggregate dynamic condition, we know that her position of momentary equilibrium must be the mean of the positions belonging to the various particles displaced; and we may assume, with a close approximation to the truth, that this is the position which would place her axis of equilibrium, or her masts, at right angles to one of the wave sub-sturfaces of equal pressure.

The sub-surface of equal pressure through the centre of gravity of a ship's displacement may be regarded in theory as a sufficiently close approximation to the effective wave surface; and it follows that when a ship deviates from the normal to this surface the effort by which she endeavours to conform to it depends upon the momentary angle of deviation in the same manner as her effort to assume an upright position when inclined in still water depends on the angle of inclination. Hence her stability or effort to become vertical in still water, measures her effort to become normal to the effective wave surface in wave water. The equations of motion for a floating body, oscillating in still water, which had previously been investigated, could therefore be applied to undulating water by introducing the condition that the position of equilibrium changed with the direction of the wave slope. Mr. Froude was not able, at first, to solve the resulting equation by adopting the trochoidal hypothesis. He therefore substituted the curve of sines for the trochoid, which gave him a form of equation he could deal with. Prof. Rankine afterwards solved the equation obtained by using the trochoid, but the results agreed with those arrived at by Mr. Froude under the conditions to which the investigations applied.

The assumptions made in order to adapt the problem to mathematical treatment were ( $\mathrm{I}$ ) that the ship is rolling passively in the trough of the sea; (2) that she is exposed to a regular series of similar waves; (3) that the waves are so large as compared with the ship that she may be assumed to accept the motion of the part of the wave she displaces ; 4 ) that the variations of apparent weight may be neglected in comparison with the actual weight; $(5)$ that the ship is of such a form as to make her still water oscillations isochronous-this being approximately the form of the old line of battle ships; and (6) that the rolling is unresisted-the effects of resistance in modifying the motion being separately considered.

The equations thus obtained by Mr. Froude, representing. the oscillations of a ship among waves as compared with those performed in still water, are most interesting; but our space will not admit of giving a full analysis of them. Their general character may, however, be appreciated if we call attention to some of their most striking features.

One critical case is that of a ship rolling among waves, whose periodic time synchronises with her own time of oscillation. It may be readily deduced from Mr. Froude's fundamental equations that, if it were not for the resistance to rolling caused by surface friction and form, a ship placed broadside on to waves which have her own periodic time, must ultimately roll completely over, however small the wave may be. It is not uncommon to find the length of a half-wave ten times the height. Such waves would increase the angle of roll by $14^{\circ} 1^{\circ}$ at each inclination, so that six successive impulses, or three com- plete waves, passing a ship would produce almost a complete overset. Though this conclusion requires to be greatly limited by introducing the element of resistance, it is obvious that such synchronism of wave-period and ship's-period must produce most formidable effects. Mr. Froude produced the result thus indicated by his theory by direct experiment with floating bodies of such form as would give approximate cases of unresisted rolling. He immersed a sphere to two-thirds of its radius; a prolate spheroid to about the same proportion of its major axis ; and a body like a flattened orange was wholly immersed, having only a very narrow neck projecting from it above the water-level, like the stem of a hydrometer. By an ingenious arrangement for regulating the period of the waves it was found that, when the oscillations of the floats and the wave-period were made to synchronise, all the floats were upset after the transit of a very few waves, while a very small change in the natural period of one of the floats, made by slightly altering the position of its centre of gravity, made its behaviour plainly exceptional as compared with the two others. It now refused to be completely overset by the series of waves which would upset the two others almost at the same moment, though it was itself overset by a series slightly quickened or retarded according as its own period was quickened or retarded by the altered position of its centre of gravity, the other two being at the same time released from all danger of capsizing.

Another critical case is when the ship possesses infinite stability; or an infinitely small radius of gyration or moment of inertia. This is not a practical possibility, but is noticed on account of the indication it gives of a ship's tendencies according to the degree in which it may possibly be approached. In this case the ship will be perfectly quick in her movements and will follow precisely the slope of the wave. The movements of a flat board laid on the water are a practical illustration of this condition. The periodic time of such a board may be practically treated as $=0$; and if a ship could be so constructed as to fulfil this condition, there might be some wisdom in attempting it. It is impossible, however, to construct a ship that will even approximately fulfil this condition, and as an approach to it could only be effected by giving her the greatest possible stability, she would only the oftener meet waves with which she would synchronise and experience the evil consequences of that condition.

One other critical case, the conditions for which are deducible from the equation of rolling motion, is that in which a vessel exposed to a series of waves performs her oscillations not in her own period but in that of the somewhat different wave period. In this case the still water oscillations would not synchronise with the wave period; but a relation subsists which enables the increasing slope of each wave to just counteract the growing inclination of the ship. At the wave hollow and crest a ship under such conditions would be upright: and she would reach her greatest inclination to the vertical when she was in an intermediate position upon the greatest slope of the wave. She would roll so that her masts would always lean towards the wave.

A general feature of the theory as deduced from the equation of rolling motion is that when the natural time of oscillation of a ship and the wave period do not synchronise, and when the rolling has not become permanent, the ship's oscillations will pass through phases analogous to the action of a pendulum when subjected to a series of impulses partially synchronous with its own excursions; and, as we have seen, this deduction is in accordance with the observed phenomena of rolling.

The results given by Mr. Froude's equation for unresisted rolling give, so far as character is concerned, a generally correct view of what actually occurs. But, quantitatively, the angles of oscillation indicated are 
largely in excess of the truth. A most important practical circumstance is left entirely out of account in the equation, viz., the fact that the oscillations are performed in a resisting medium. The laws of resistance to rolling are not sufficiently well understood to enable their modifying effect to be introduced into a general equation, and a direct and theoretical solution of the question thus attempted. $\mathrm{Mr}$. Froude has shown, bowever, how in the case of any individual ship an approximate solution may be obtained with certainty by help of data derived from a single experiment with the ship herself, or even with a carefully made model of her, tried in still water. He has also pointed out that if a well-selected series of such experiments were tried for ships of different forms, and the results tabulated, the series of corresponding solutions would enable him to determine, as if $d$ priori, what modifications the results of his equation would require for any ship whatever.

The resistance which a ship experiences in oscillating through a given angle among waves is practically the same as it would be if she were performing an oscillation of the same range in still water. If she is set rolling in still water the resistance will rapidly bring her to rest when the force which caused ber to roll ceases to act. In the same way, when rolling among waves, the same resistance will reduce the angles of oscillation. It is this circumstance which prevents dangerous angles being reached in the critical case of synchronism, and which, at all times, fixes the limiting position to which a ship will roll. The action of the waves in increasing the angle of roll is Lalanced by the opposing tendency of resistance to reduce it. The aggregate resistance of a ship to rolling is made up of three parts: (I) That due to the friction of the fluid in moving over the skin of the ship; (2) the direct resistance of the keel and fine parts at the ends to being pushed through the water at right angles, or nearly so, to their planes; and (3) what Mr. Froude calls the "wave-making function," or the element of resistance caused by the successive displacements of fluid in rolling which affect the surface as waves, and travel away from the ship, thus abstracting from her the energy they embody.

The aggregate resistance to rolling being of such a character, and containing elements which vary in different ways with the rate of motion of the surface of a ship's bottom, its exact computation in a particular case would be very difficult. Mr. Fronde has, however, done much towards enabling a sufficiently close approximation to be arrived at by direct calculation. For practical purposes, however, Mr. Froude's original suggestion of determining the amount of resistance by still water experiments is employed. A ship is set rolling in still water, and upon reaching an angle of sufficient magnitude, she is allowed to come to rest under the action of resistance only. The rate of extinction of her range of oscillation is continuously noted and registered in the form of a curve. It will be obvious that the difference in amplitude of two successive swings measures the aggregate effect of the resistances operating upon the ship, and that by means of a complete curve of extinction a measure of the resistance opposed by her to rolling among waves may be obtained.

This is, necessarily, but a general description of $\mathrm{Mr}$. Froude's theory, and does not include many details and some collateral matter which are of importance in making it quite compleie; but it will serve to give an indication of its broad features.

Mr. Froude's theory has met with general acceptance as being a sufficiently close approximation to a correct expression of the laws of motion of a floating body among waves. Some of the departures from absolute accuracy contained in the assumptions necessary to the formation of the theory, such as that wave-water is continually changing its form, and a ship being a rigid body, cannot therefore, strictly speaking, be said to accept the dynamical conditions of the displaced fluid as it accepts the statical conditions of its still-water displacement; and the objection that a sub-surface of equal pressure does not correctly represent the effective wave surface have been criticised; but the criticisms have only shown that the errors thus involved are very small in amount and have no appreciable effect upon the general results of the theory. Mr. Froude has always insisted himself upon the existence of imperfections in his theory, but he has constantly been at work upon their removal, and upon the determination of the tangible amount of error they introduce into the ultimate result.

Mr. Froude's work was undertaken at first with a practical object, on account of Mr. Brunel's requirements, and it has already accomplished great practical results. It has led to the conclusion that ships of long periods are least likely to meet with waves which will cause them to roll heavily, and that the rolling of a ship can be greatly reduced by means of deep-bilge keels. These ideas have been extensively acted upon in H.M. Service. Our heavy armour-clads and other ships of war have been designed in accordance with this theory, so as to have great steadiness at sea, and in 'many special cases their properties have been determined by Mr. Froude beforehand, so that instead of working in the dark the Admiralty have known what behaviour to expect from a ship after she is built. The effect of bilge-keels in extinguishing rolling was clearly shown by Mr. Froude in $187 \mathrm{r}$, with a model of the Devastation. Without bilge-keels the model performed $31 \frac{1}{2}$ complete oscillations before coming to rest in still water, after being inclined to an angle of $8 \frac{1}{2}^{\circ}$, but with a bilge-keel equivalent to 36 inches in depth on the full scale of the ship, she came to rest in eight oscillations, and with a bilge keel double this depth she came to rest in four oscillations. The same model when tried among waves which capsized her when no bilge keels were fitted only rolled to an angle of $13 \frac{1}{2}^{\circ}$ with 3 feet bilge keels, and to $5^{\circ}$ with 6 feet bilge keels. These experiments showed conclusively the effect of bilge keels in limiting the range of oscillation among waves.

The importance of bilge keels was further tested by comparative trials conducted by $\mathrm{Mr}$. Froude off Plymouts in 1872 between H.M. ships Greyhound and Perseusboth of the same class-the former having bilge keels and the latter being without them. Mr. Froude also conducted an elaborate series of experiments on board the Devastation at sea in 1873 and 1875 , and, by means of a most ingenious machine of his own construction, obtained continuous automatic records of her behaviour. These records showed (I) the relative inclination of the ship and the effective wave slope at any instant; (2) the inclination of the ship to the vertical at any instant; and (3) the period of oscillation of the ship at any time, that is, the number of seconds occupied in completing the roll from port to starboard, and vice rersâ. From the diagrams upon which (I) and (2) are traced in the form of curves Mr. Froude deduced, as a differential result, the period and angle of slope of the effective wave surface at any instant, thus determining it with a much greater degree of exactness than that with which the form of a surface wave could be ascertained.

Mr. Froude was constantly checking and correcting his theoretical results by the aid of experiment, and though he bad succeeded in fully establishing the true theory of the rolling of ships among waves, he did not rest satisfied with his success, but had recently made arrangements at his experimenting tank at Torquay for proceeding with further important lines of investigation by means of observations upon the behaviour of models in waves mechanically generated.

We have only dealt with Mr. Froude's labours in connection with the question of the rolling of ships in this number, and must reserve a description of his investigations into the laws of resistance and propulsion, which are at least of equal importance, for another occasion. (To be continued.) 